\title{
Letters
}

\section{A Dual-Polarized Slot Antenna for Millimeter Waves}

Goutam Chattopadhyay and Jonas Zmuidzinas

Abstract-We describe a new dual-polarized slot antenna to be used with quasi-optical devices such as superconductor-insulatorsuperconductor (SIS) mixers at millimeter and submillimeter wavelengths. The radiation and impedance characteristics of the antenna were obtained from a moment-method calculation. The antenna has an excellent radiation pattern, a low impedance, wide bandwidth, and low cross polarization.

Index Terms-Slot antennas.

\section{INTRODUCTION}

The twin-slot antenna on a hyperhemispherical substrate lens has been shown to be a good structure for single polarization receivers [1]. We propose a four-slot structure as shown in Fig. 1, which is basically an extension of the twin-slot design for dual polarization. The structure was analyzed using a numerical momentmethod analysis technique in the Fourier domain similar to that described by Kominami et al. [2].

\section{Method of Moments AnAlysis}

As usual, the substrate lens is treated as a semi-infinite dielectric slab. This is a very good approximation since, in practice, reflections at the lens surface can be largely eliminated by using an antireflection coating [3]. We consider the case in which the horizontal slots are excited symmetrically (Fig. 1). The field distribution in the slots can be intutively obtained from symmetry considerations. In particular, the field distribution in the vertical slots must be antisymmetric and, therefore, the voltage at the orthogonal ports must vanish. We define $z$ axis to be normal to the antenna plane, the length and width of the slots as $L$ and $W$, and the slot separation as $S$. We also define two vectors on the $x-y$ plane $\overrightarrow{k_{t}}=\left(k_{x}, k_{y}, 0\right)$ and $\overrightarrow{p_{t}}=\hat{z} \times \overrightarrow{k_{t}}=\left(-k_{y}, k_{x}, 0\right)$. The admittance kernel, which relates the Fourier transforms of the electric fields and surface currents on the antenna plane is a tensor under rotations of the $x-y$ plane, and can be written as

$Q_{\alpha \beta}\left(\overrightarrow{k_{t}}\right)=\frac{1}{Z_{0}}\left[\left(\frac{\epsilon_{1} k}{\gamma_{1}}+\frac{\epsilon_{2} k}{\gamma_{2}}\right) \delta_{\alpha \beta}-\left(\frac{1}{k \gamma_{1}}+\frac{1}{k \gamma_{2}}\right) p_{\alpha} p_{\beta}\right]$

where $\delta_{\alpha \beta}$ is the Kronecker delta function $\left(\delta_{\alpha \beta}=1\right.$ for $\alpha=$ $\beta$ and $\delta_{\alpha \beta}=0$ for $\alpha \neq \beta ; \alpha, \beta=x$ or $\left.y\right), p_{\alpha}$ is a component of the vector $\vec{p}_{t}, k=\omega / c$ is the magnitude of the wave vector in free-space, $Z_{0}=377 \Omega$ is the impedance of free-space, $\epsilon_{1}$ and $\epsilon_{2}$ are the dielectric constants above $(z>0)$ and below $(z<0)$ the antenna plane, and the $z$ component of the wave vector in the two half spaces are $\gamma_{s}^{2}=\epsilon_{s} k^{2}-k_{x}^{2}-k_{y}^{2}$ with the conditions $\operatorname{Im}\left(\gamma_{1}\right) \leq 0$ and $\operatorname{Im}\left(\gamma_{2}\right) \geq 0$. The electric fields in the slots are expanded in a

Manuscript received August 26, 1997; revised November 10, 1997.

G. Chattopadhyay is with the Department of Electrical Engineering, California Institute of Technology, Pasadena, CA 91125 USA.

J. Zmuidzinas is with the Downs Laboratory of Physics, California Institute of Technology, Pasadena, CA 91125 USA.

Publisher Item Identifier S 0018-926X(98)03417-6.

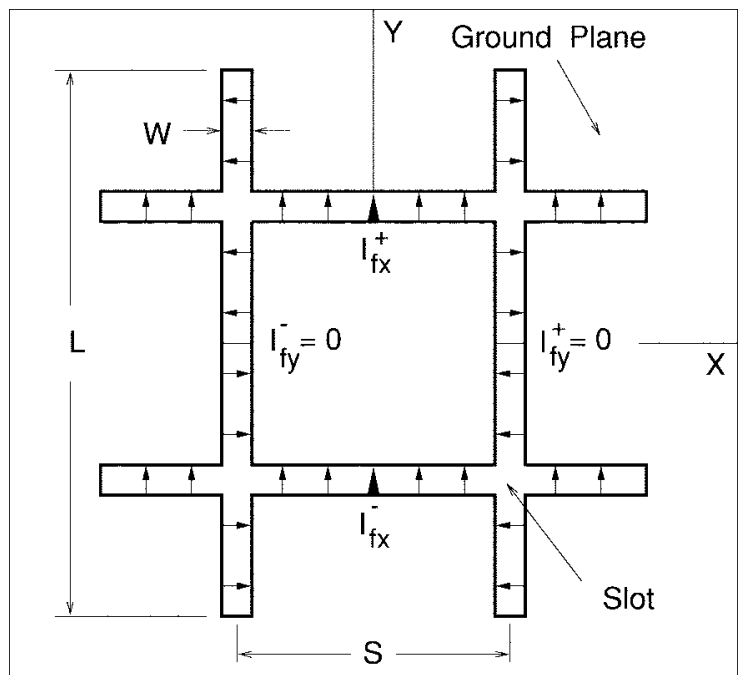

Fig. 1. Proposed antenna structure. The four feed points are at the centers of the slots. The horizontal slots are symmetrically excited $I_{f x}^{+}=I_{f x}^{-}$and the vertical slots are not excited $I_{f y}^{+}=I_{f y}^{-}=0$.

set of basis functions $f_{x i}, f_{y i}$

$$
\begin{aligned}
& E_{x}(x, y)=\sum_{i}\left[V_{x i}^{+} f_{x i}\left(x, y-\frac{S}{2}\right)+V_{x i}^{-} f_{x i}\left(x, y+\frac{S}{2}\right)\right] \\
& E_{y}(x, y)=\sum_{i}\left[V_{y i}^{+} f_{y i}\left(x-\frac{S}{2}, y\right)+V_{y i}^{-} f_{y i}\left(x+\frac{S}{2}, y\right)\right]
\end{aligned}
$$

where $V_{x i}^{ \pm}$and $V_{y i}^{ \pm}$are the unknown amplitudes. The electric fields are Fourier transformed and then multiplied by the admittance kernels to yield the surface currents

$$
J_{\alpha}\left(\overrightarrow{k_{t}}\right)=\sum_{\beta} Q_{\alpha \beta}\left(\overrightarrow{k_{t}}\right) E_{\beta}\left(\overrightarrow{k_{t}}\right)
$$

Using the basis functions as testing functions (the Galerkin method), we get the following matrix equations for the amplitudes $V_{x i}^{ \pm} V_{y i}^{ \pm}$:

$$
I_{x i}^{+}=\sum_{j}\left[V_{x j}^{+} Y_{x x, i j}^{++}+V_{x j}^{-} Y_{x x, i j}^{-+}+V_{y j}^{+} Y_{y x, i j}^{++}+V_{y j}^{-} Y_{y x, i j}^{-+}\right] .
$$

Similar equations for $I_{x i}^{-}$and $I_{y i}^{ \pm}$can be derived in the same way. The admittance matrix elements are obtained from the admittance kernels and the Fourier transforms of the basis functions through the numerical evaluation of the integrals of the form

$$
Y_{x x, i j}^{ \pm \pm}=\frac{-1}{(2 \pi)^{2}} \iint_{-\infty}^{\infty} Q_{x x} \tilde{f_{x i}} e^{ \pm j k_{y} S / 2}\left(\tilde{f_{x j}} e^{ \pm j k_{y} S / 2}\right)^{*} d k_{x} d k_{y}
$$

where $\tilde{f_{x i}}$ is the Fourier transform of $f_{x i}(x, y)$ and the $(*)$ denotes complex conjugate. We have five such independent elements with $Y_{x x}^{++}=Y_{x x}^{--}, Y_{y y}^{++}=Y_{y y}^{--}, Y_{x x}^{+-}=Y_{x x}^{-+}, Y_{y y}^{+-}=Y_{y y}^{-+}$, and $Y_{x y}^{++}=Y_{x y}^{-+}=Y_{y x}^{++}=Y_{y x}^{+-}=-Y_{x y}^{--}=-Y_{x y}^{+-}=-Y_{y x}^{--}=$ $-Y_{y x}^{-+}$. We use entire domain basis (EDB) functions as used by Zmuidzinas and LeDuc [1] for the horizontal slots; for the vertical slots we use antisymmetric triangular basis functions given by

$$
A_{n}(x ; d)=T\left(x-x_{n} ; d\right)-T\left(x+x_{n} ; d\right)
$$




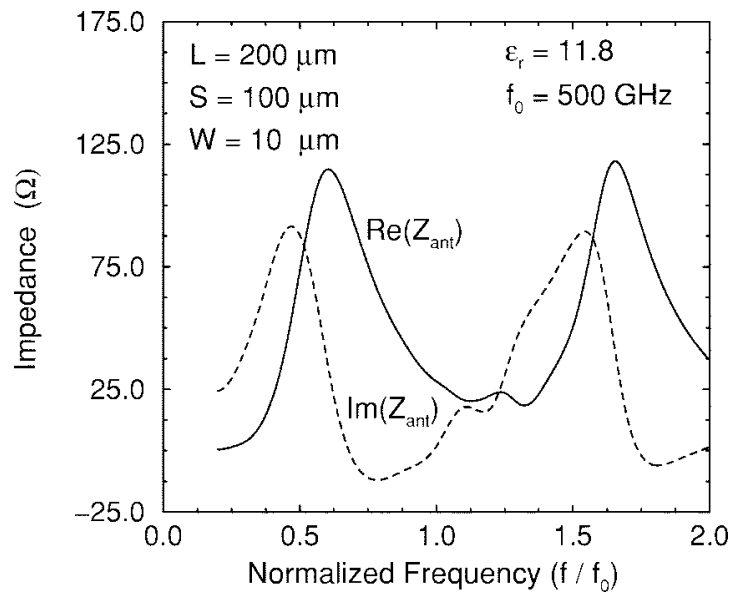

Fig. 2. Antenna impedance as a function of frequency for the cross-slot antenna on a semi-infinite silicon dielectric substrate.

where $x_{n}=n d ; n=1,2, \ldots, n_{\max } ; d=L /\left[2\left(n_{\max }+1\right]\right.$, and $T(x, d)$ is a triangular function extended over $(-d, d)$. The equations $I_{x i}^{ \pm}$and $I_{y i}^{ \pm}$are overlap integrals between the feed currents in the slots and the basis functions. Thus, $I_{x i}^{+}=I_{x i}^{-}=I_{f x}$ - the feed current-since the horizontal slots are excited symmetrically-and $I_{y i}^{+}=I_{y i}^{-}=0$ - since the vertical slots are not excited. Inverting the admittance matrix we will get the impedance matrix as in ([1]) and for symmetric excitation, the impedance of each horizontal slot will be

$$
Z_{\mathrm{ant}}^{+}=\sum_{i, j} Z_{x x, i j}^{++}+\sum_{i, j} Z_{x x, i j}^{-+}
$$

The mode amplitudes $V_{x i}^{ \pm}$and $V_{y i}^{ \pm}$are calculated from the impedance matrix and the feed currents in the same manner as in [1]. These mode amplitudes allow the electric fields in the slots to be calculated in the Fourier domain, which are used to obtain the copolarized and crosspolarized radiated fields. For cross-polarization calculations, the third definition given by Ludwig [4] is used.

\section{RESULTS}

We calculated a cross-slot antenna with dimensions $L=200 \mu \mathrm{m}$, $S=100 \mu \mathrm{m}, W=10 \mu \mathrm{m}$, which is our "standard" geometry for a $500-\mathrm{GHz}$ antenna on a silicon $\left(\epsilon_{r}=11.8\right)$ substrate. Typically, six basis functions of each type (EDB and triangular) are used in the analysis. The calculated impedance as a function of frequency is plotted in Fig. 2, and, in general, is quite similar to the twin-slot case. The $1-\mathrm{dB}$ impedance bandwidth for matching to a $30-\Omega$ resistive load was found to be about $200 \mathrm{GHz}$. The main beam efficiency (power in the main lobe to the first null) is quite high- $88.2 \%$ (see Table I). Contour plots for the co-polarized and cross-polarized power pattern radiated into the dielectric are shown in Fig. 3. The peak in cross-polarization was found to be $24 \mathrm{~dB}$ below the copolarized peak power. The hyperhemispherical lens on which the antenna will be placed will affect the cross-polarization response because the transmission through the air/dielectric interface at the lens surface depends on the polarization and angle of incidence. However, our experimental wire-grid polarizer measurements on a $500-\mathrm{GHz}$ superconductor-insulator-superconductor (SIS) receiver using a twinslot antenna show that the total cross-polarization is no more than $-20 \mathrm{~dB}$, indicating that the effect of an antireflection coated lens on the cross-polarization is quite low.

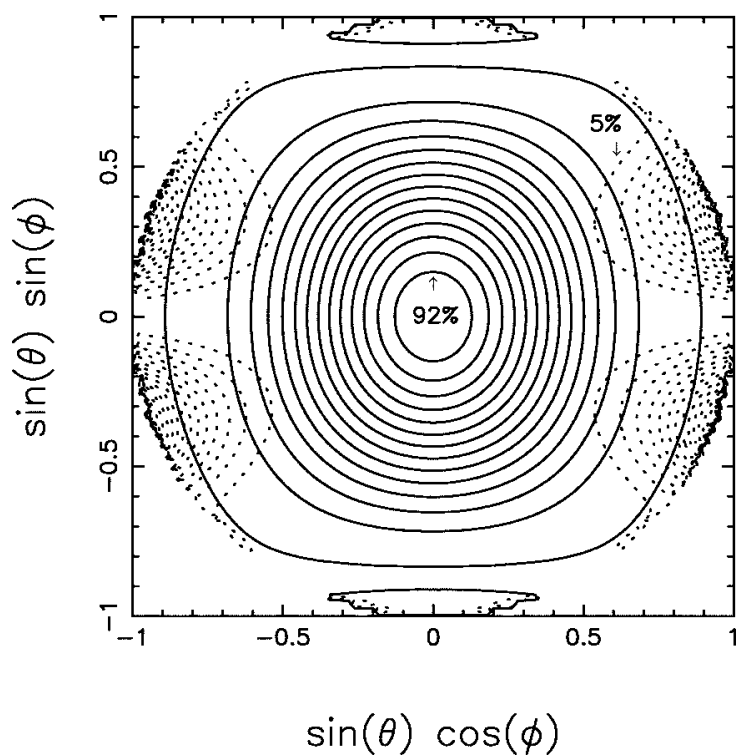

Fig. 3. Contour plots of the calculated co-polarized and cross-polarized power pattern radiated into the dielectric by the antenna. The solid lines are for the co-polarized power and the dotted lines are for the cross-polarized power. The dimensions of the slot are the same as in Fig. 2. For the co-polarized power, the contours are linearly spaced from 0.5 to $92 \%$ of the peak co-polar power in increments of 7\%; and for the cross-polarized power, the contours are linearly spaced from 5 to $95 \%$ of the peak cross-polar power in increments of $5 \%$. The quantities $(\theta, \phi)$ are the usual polar angles with respect to $\hat{z}$.

TABLE I

ANTENNA Results

\begin{tabular}{|c|c|c|}
\hline & E-plane & H-plane \\
\hline HWHM & $26^{\circ}$ & $23^{\circ}$ \\
\hline Phase $-3 \mathrm{~dB}$ & $0^{\circ}$ & $-2.9^{\circ}$ \\
\hline \multicolumn{3}{|l|}{ Power lost to: } \\
\hline \multicolumn{3}{|c|}{ 1) Cross-polarized field in dielectric $=0.6 \%$} \\
\hline \multicolumn{3}{|c|}{ 2) Sidelobes in dielectric $=1.3 \%$} \\
\hline \multicolumn{3}{|c|}{ Power radiated backward into "air" $=9.9 \%$} \\
\hline \multicolumn{3}{|c|}{ Main beam efficiency for co-pol beam $=88.2 \%$} \\
\hline
\end{tabular}

\section{CONCLUSION}

The cross-slot antenna appears to be an excellent candidate for millimeter and submillimeter quasi-optical devices. The impedance, radiation pattern, and bandwidth of the cross slot are very similar to those of the twin slot. In comparison to the dual-polarized slot-ring antenna [5], the cross slot offers superior radiation characteristics (the radiation pattern of the cross slot is narrower since it is physically larger) at the expense of four feed points instead of two.

\section{REFERENCES}

[1] J. Zmuidzinas and H. G. LeDuc, "Quasi-optical slot antenna SIS mixers," IEEE Trans. Microwave Theory Tech., vol. 40, pp. 1797-1804, Sept. 1992.

[2] M. Kominami, D. M. Pozar, and D. H. Schaubert, "Dipole and slot elements and arrays on semi-infinite substrates," IEEE Trans. Antennas Propagat., vol. AP-33, pp. 600-607, June 1985.

[3] M. C. Gaidis, H. G. LeDuc, M. Bin, D. Miller, J. A. Stern, and J. Zmuidzinas, "Characterization of low-noise quasioptical SIS mixers for the submillimeter band," IEEE Trans. Microwave Theory Tech., vol. 44, pp. 1130-1139, July 1996.

[4] A. C. Ludwig, "The definition of cross polarization," IEEE Trans. Antennas Propagat., vol. AP-21, pp. 116-119, Jan. 1973.

[5] S. Raman and G. M. Rebeiz, "Single- and dual-polarized millimeterwave slot-ring antennas," IEEE Trans. Antennas Propagat., vol. 44, pp. 1438-1444, Nov. 1996. 